\title{
Research on the Application of CAD Auxiliary Intelligent Technology in Sports
}

\author{
Wenhong Wang (iD \\ Department of Physical Education, Jinling Institute of Technology, Nanjing 211169, China \\ Correspondence should be addressed to Wenhong Wang; wwh@jit.edu.cn
}

Received 3 November 2021; Revised 29 November 2021; Accepted 10 December 2021; Published 17 January 2022

Academic Editor: Qiangyi Li

Copyright ( $\odot 2022$ Wenhong Wang. This is an open access article distributed under the Creative Commons Attribution License, which permits unrestricted use, distribution, and reproduction in any medium, provided the original work is properly cited.

\begin{abstract}
This paper mainly combines CAD auxiliary intelligent technology and image processing technology, by combining the experience and creativity of sports coaches and sports researchers, and using modern technology, it can design and efficiently study sports quickly, conveniently, and efficiently and uses advanced high-tech technology to replace the traditional relatively backward artificial design method of sports. In the field of sports, in-depth research and scientific research and feasibility analysis can be conducted, so as to realize the design of CAD auxiliary intelligent technology system for sports competitive movements. Finally, the experimental results show the CAD auxiliary intelligence technology in sports to verify the feasibility and have practical value.
\end{abstract}

\section{Introduction}

At present, there are more sports events in a set of movements selected by the contestants. The degree of innovation, demonstration quality, and change of difficulty design in the sports competition process will all affect the results given by the judges [1-3]. In the past, artificially designed sports had the disadvantages of long action design cycle, insufficient innovation of creation content, and impossible drill in advance, which seriously affected the improvement and longterm development of the sports competition level. The use of CAD-assisted intelligent technology in sports and can make the computer rapid and accurate processing ability can be improved; for experienced coaches and athletes, with the advantages of rapid, accurate, and reliable CAD software, auxiliary simulation training can be carried out. College physical education has realized the transformation from pure teaching to comprehensive training. Educational methods have also changed from traditional teacher and student experience to technical training, and the forms of competition are also diversified. In other words, CADassisted intelligence technology has been widely used in all walks of life. The specific applications of high school sports are as follows. The details are shown in Figure 1. The application of CAD-assisted intelligent technology in sports conference management education and training provides a new way of learning for sports learners. The application of CAD-assisted intelligent technology in some sports, entertainment, etc., greatly reduces sports such as skiing, racing, and other dangerous sports. It can be trained indoors through CAD-assisted intelligent technology. Accidents such as avalanches and collisions should be avoided. CADassisted intelligence technology has achieved a realm that is difficult to achieve by other objective factors. As an athlete's trainer, to a certain extent, the player's winning rate has been improved. In some countries and regions, it is difficult for training and athletes to reach each other's level. A detailed analysis of sports is carried out to explore the problems and shortcomings in the exercise process, so as to formulate training and improvement plans in the later period. In the traditional sports posture real-time analysis system positioning accuracy filter algorithm research process, usually two-dimensional map is used to describe the sports environment of the sports posture real-time analysis system, but because the two-dimensional map can only describe the relatively high level of environmental plane information, it cannot provide relatively complete data information. At present, the requirements of sports require sports companies to be able to solve a one-stop service system, to provide strong support for life, and to obtain the highest economic 
benefits for the entire sports. This is the overall goal of the work of sports companies. Real-time posture analysis in sports has an important position. A good posture real-time analysis system can speed up the flow of sports, reduce costs, ensure the proper operation of services, and realize the effective management and use of resources.

In the process of sports, CAD auxiliary intelligent technology not only needs to further improve the scientific sports training but also will greatly improve the level of sports competition. The application of this technology to real life has gradually become an important problem that sports coaches and athletes will face. Finally, the experimental results show that the sports application analysis based on CAD auxiliary intelligent technology can effectively improve the sports level of sports athletes.

\section{Study Purpose}

In the process of sports competition, many sports are the competitions independently selected by the athletes. With the continuous improvement of the sports competition level, competitive competitions have also put forward higher requirements in terms of training quality and difficulty coefficient, and the action innovation of the athletes has also improved the requirements [4-8]. If athletes can master a kind of unique design, high movement difficulty coefficient, more movement changes, is the key to win in sports competition. In sports, it still stays in the previous artificial innovation design stage, mainly with long design cycle, low efficiency, and low quality. Creation from narrow, unless the athletes themselves experiment, cannot simulate the demonstration movement; this operation, completely relying on the original manual creation, has fallen behind the rapid development of modern sports movement, to some extent not only limited the rapid progress and development of difficult and new movements but also seriously affected the progress of the sports level.

CAD auxiliary intelligent technology is a kind of hightech technology in the field of electronic information, applied to sports, can make the sports innovative, can also quickly solve modern science and technology to replace the traditional artificial design method, realize the sports total design autonomy, and is of great significance.

\section{Study Method}

By using CAD-assisted intelligent technology and computer image processing technology and effectively combining the evaluation and actual combat experience of sports coaches or auxiliary sports action designers, a computer-aided design system that can be used for sports athletic actions is constructed, using a human-computer interaction. The model completes the design of the technical image and image movement of the sports competitive human body. CADassisted intelligence technology is also a kind of back propagation technology, which is the most widely used at present. The main idea of this technology is to use the theoretical knowledge of gradient search to use the Internet to achieve the minimum value of the error value between the

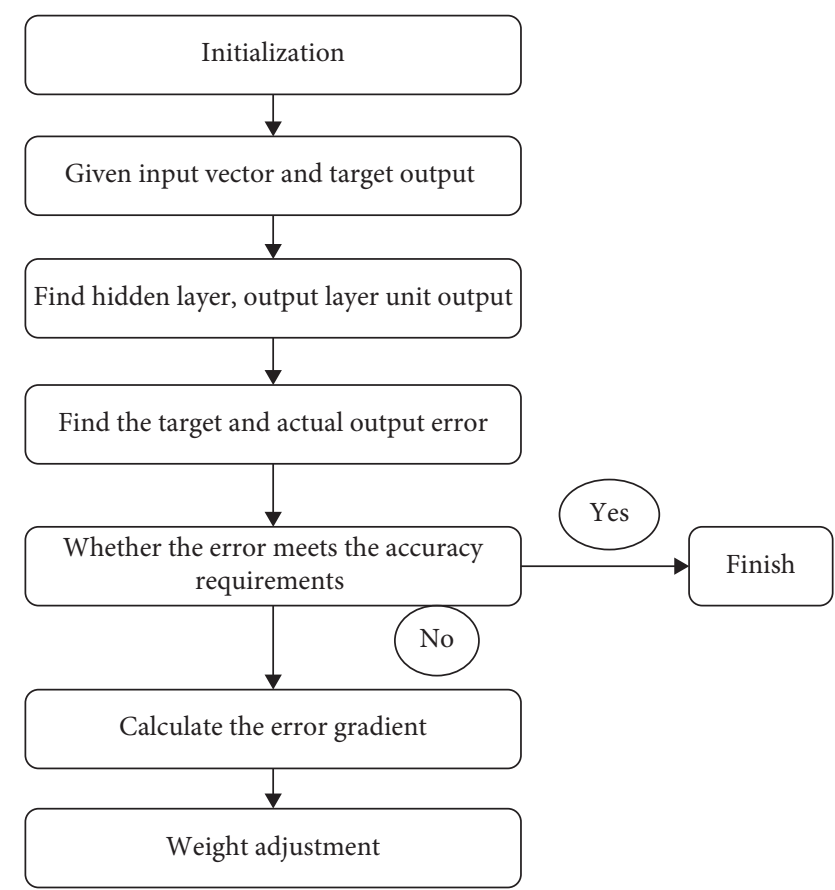

FIgURE 1: CAD auxiliary intelligent calculation flowchart.

actual data output and the expected value output. It can input the variable $\mathrm{Xi}$, and the intermediate node will have an impact on the output node. According to the complex nonlinear conversion process, the output variable $Y_{k}$ is generated [9-11]. If the output value of the response variable and the built model variable quality inspection is greater than the set standard error, then the built model needs to reset the weight of each layer; until the error value is less than the set value, the sports training will be suspended. The calculation flowchart of CAD-assisted intelligence is shown in Figure 1.

3.1. The Composition of the System. The system mainly includes information input equipment, host (computer or workstation), image processing system, graphics library, graphics generation system software, and output equipment (Figure 2). Select the CAD auxiliary intelligence technology. This is a relatively perfect three-dimensional motion creation software combined with the function of the SGI workstation and adopts multimedia technology, motion modeling and change technology, and dynamic simulation technology. The requirements can be met in the design of competitive sports image and analysis processing, and the functional module is shown in the attached figure.

3.1.1. Hardware Environment. According to the host type of computer, it can be divided into microcomputer and workstation. Microcomputer generally use models above $586 / 133$ models, the memory capacity greater than $16 \mathrm{MB}$, hard disk greater than 1.2 GB, high resolution 20 inch display, and speed processing board card, corresponding 8XCD drive, decompressing card, sound card and output equipment as a scanner, digital image input module, camera, 


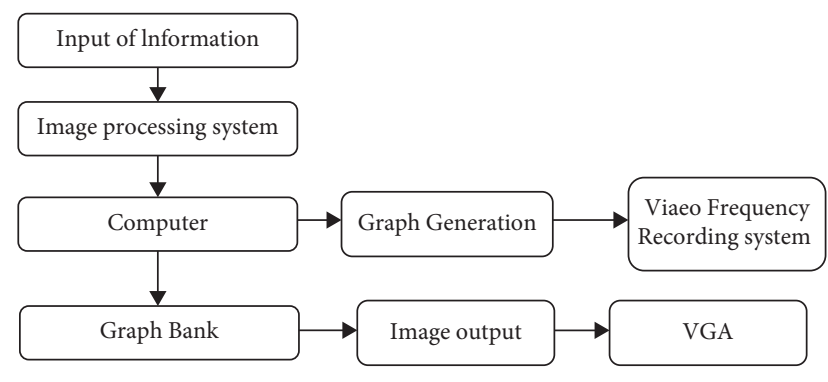

FIGURE 2: System composition and workflow.

display screen, mouse, etc. The output equipment is mainly image printer, painting instrument, etc., and CAD auxiliary intelligent technology software is saved as the form of image generation. There are three software. First, the action images displayed on the screen are mainly displayed on the screen; the other is the sports video output. The digital signal corresponding to the calculation generated image can be converted into video signals to be recorded on the device and display on the TV screen. Third is the rubber coil output.

The video conversion board will be loaded to the SGI workstation, for example, the Genlock board, the microcomputer into the TARGA board. For the computer to control the video recording device, a VTR controller, such as the VLAN, is required to achieve automatic control of each frame.

3.1.2. Software Environment. Microcomputer classes can select 3D studio. From Autodesk Inc (3.3), original design software is characterized by simple operation and no professional knowledge and is composed of five functional modules. That workflow is shown in Figure 3.

The working process of each section depicts the plane drawing with 2Dsharper, makes three-dimensional action drawing with 3DLflater, and sets the action setting (place, machine, equipment, etc.) with 3DEditor. Later, the effect processing with Material Editor finally enters the keyflamer for the button screen editing of the key action point [12-15]. After completing these five parts, the system will automatically generate the transition of the intermediate connection motion, complete the movement, rotation, subversion, and change of the action, and develop two software for the special action.

The host chose the SGI workstation, and the corresponding software was elected SOFTTIMAGE 3D, which is an advanced software system that would combine its functionality and perfect 3D action creation. SOFTTIMAGE 3D uses multimedia technology, sports styling and change technology, and dynamic simulation technology. Together with the competitive action computer support system composed of SGI workstation and SUN-VICOM image processing system, the software is required to analyze the speed of action images of sports technology and the effect of design prompt action.

3.2. New Action Demonstrations and Modifications. A new action image generated in the image generation software of the design system provides such an operational tool by the general software system when changing the image size or

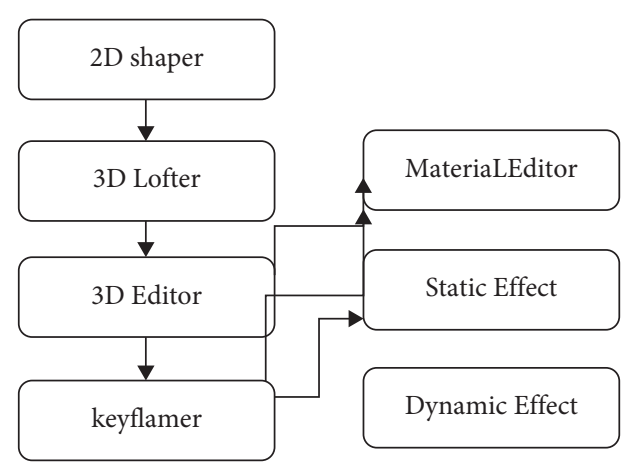

FIgURe 3: Workflow chart of Dstudio.

changing the image.To show the action effect on the picture, first use the dynamic presentation of the outline map. Although it is easy to correct, the authenticity of the effect is not enough, so the reduced action is to be demonstrated through the image operation and corrected before the formal action.

\section{CAD Is Assisted in Intelligent Design Model Analysis}

4.1. The CAD-Assisted Intelligent Object Model. In order to correctly display and store the training knowledge of various sports projects, the system design adopts the target software engineering method (OOSE), with objects as "target software," the sports training knowledge of organizing and managing sports projects for units.

In this paper, sports objects are model constructed using CAD-assisted intelligence technology. Among them, the attribute area is mainly the STOM attribute, namely, the basic data information corresponding to STOM. Methods mainly include the basic operation of the STOM, namely, the STOM method and the corresponding action. Association regions include various relationships between STOM. The case field contains different types of available cases for STOM.

4.2. CAD-Assisted Intelligent Technology Intelligent Method and Dynamic Model. According to the sports training program, the physical training tasks of sports training can be processed using CAD-assisted intelligent technology, and from the system database, the reference items containing sports sports training and the basic knowledge of sports training can be searched. According to the individual parameter characteristic value of the sports training objectives, the reference sports training can be manually processed and intervened, the new sports training content is planned, the sports training is improved and promoted, and the sports goals have been achieved. The system dynamic model is as follows.

4.2.1. Assessment of Sports Training Quality for Athletes. According to the type of sports, characteristics, and competitive requirements, from the perspective of sports requirements on the athletes 'physical situation, sports quality, 
sports ability, sports strategy, sports wisdom, sports psychological quality, cultural level, and their physical condition and other aspects, the sports athletes' current competitive state, competitive ability, and competitive potential of comprehensive evaluation can effectively obtain the comprehensive athletes and provide reference.

\subsubsection{Sports Training Mission Completion Planning.} Make the next stage of sports competitive training, according to the established goals, to formulate the corresponding sports training content, segmentation tasks to specific, detailed specific division of labor, and the complete sports training task divided into multiple independent and related training objectives; at each stage, sports training tasks, sports training content, and sports training items can be different.

\subsubsection{Construction of Sports Training Scheme. According to} the types of sports items, sports training stage, sports characteristics, skill requirements, competitive objectives, and sports training content requirements, this paper retrieves and screens the CAD software basic knowledge base and randomly selects the previous sports training program and the sports training program to develop the first sports training plan.

4.2.4. Adjustment of the Sports Training Plan. According to the evaluation of sports athletes at different stages, the application of CAD auxiliary intelligent technology to interpret the sports training knowledge rules can effectively improve the plan makers to adjust the sports training plans according to the actual situation, so as to facilitate reasonable control, supervise, and formulate the relevant sports training plans.

4.2.5. Implementation and Monitoring of the Sports Training Program. Athletes shall undergo comprehensive sports training according to the requirements and steps of the sports training program, and the sports training process is supervised and controlled. The various information and sports training data in sports training shall be recorded at any time to provide scientific reference for the improvement of subsequent physical exercise.

4.2.6. Sports Training Information Feedback and Effect Evaluation. Organize, compare, and analyze various data and sports training information feedback during the process of sports training, correctly evaluate the differences between sports training objectives and sports training results, and confirm whether the sports training objectives are achieved. Help the coach and summarize the successful experience and failure lessons of sports training in time so that the results of physical training are scientifically close to the goal of sports training.

4.2.7. Collection and Management of Sports Training Knowledge and Sports Training Cases. Mainly collect the data information from coaches, athletes, and auxiliary sports trainers, conduct comprehensive analysis and evaluation through relevant sports examples and sports events, and timely supplement the case base according to the relevant sports training knowledge of data analysis. The system manages the training situation and training knowledge of existing projects.

4.3. The CAD-Assisted Intelligent Technology Function Model. Sports development has steadily increased, but there are still shortcomings. The events that affect Jiangxi's international competitions and national competitions are mainly kayaking and weightlifting. This has brought unprecedented challenges to sports development. Single projects and poor performance are the limitations of sports development. This system adopts the design form of real-time analysis of three-dimensional virtual posture, which has small size, low cost, and good connection performance. It uses the strong analysis method of system positioning accuracy filtering to locate and analyze sports system data to improve the accuracy of positioning. It can be applied to the monitoring system of each line. The monitoring system can determine the detailed location of the item based on the received data, with high efficiency and high accuracy. Through many experiments, in the context of obstacles, CAD-assisted intelligent technology has high accuracy and can receive information in real time. People's requirements for quality are constantly improving, so it is necessary to improve the accuracy of positioning in different environments. This demand is the direction of future research.

The sports CAD, sports training system, is divided into a series of construction units based on the specific sports training program. The structural unit also consists of a series of functional modules. The hierarchical structure of the software is illustrated in Figure 4.

Referring to the main contents and implementation steps of the sports training control process, the sports training control parameters are comprehensively extracted according to their competitive characteristics, the experience and lessons of sports training are applied to the process of sports training, and a feature-based sports training information model is established.

The whole system is divided into auxiliary sports training and auxiliary service parts. The auxiliary sports training part is composed of the sports training subsystems of some sports projects. Each sports training subsystem is relatively independent and noninterfering with each other and includes auxiliary sports training behavior and functional modules for each sport. 


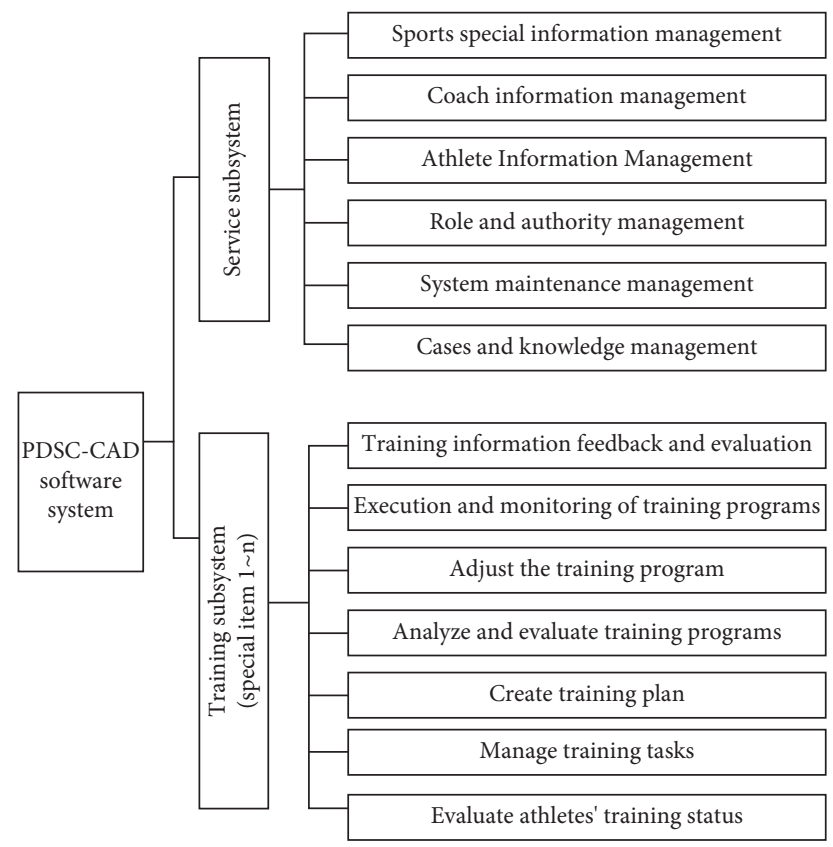

FIGURE 4: CAD auxiliary intelligent technology function model.

These subsystems can help you directly with physical training. The service subsystem consists of six service behaviors and its corresponding functional modules and mainly provides service assistance functions for the overall maintenance, operation, and management of the system.

4.4. CAD-Assisted Intelligent Technology Architecture. CAD auxiliary intelligence technology takes the dynamic management of sports training cases and sports training knowledge as the main starting point, builds the corresponding decentralized database support environment, and uses the basic data [11] such as artificial input cases and knowledge. According to the types, characteristics, competitive requirements, and the status parameters of the players, the precedents and knowledge in the box base and knowledge base are dynamically extracted, and the precedents and knowledge extracted are analyzed, diagnosed, and evaluated. At the same time, the case and knowledge will be used for the project sports training, the sports training process monitoring, the feedback information collection of the sports training process, the sports training program and the sports training process evaluation, and the integrated management of the project sports training realization.

As shown in Figure 5, the CAD-assisted intelligence technology uses the $\mathrm{B} / \mathrm{S}$ architecture. By submitting service requests to customers, you can read and extract system resources to realize data exchange, data transfer, and data sharing [12]. Servers mainly include database servers and file servers. It can be used for file management, data transmission, and system resource storage. The end user requests to the server via the client browser, performs the corresponding service on the server, and returns the execution result to the end user [13] via the browser.

\section{Experiment and Results Analysis}

5.1. Filening of Model Independent Variables. Because the correlation strength of each quality sports training index and specific project performance is different, the influence coefficient of specific project on performance design is also different. It is necessary to identify the physical training indicators that have a great impact on the special performance of the athletes. Using the historical data of long jumpers included in the General Administration of Sport of China from 2018 to 2019, the various quality sports training indicators and special achievements of long jumpers were analyzed, and the corresponding correlation coefficient was calculated. Results are shown in Table 1. As can be seen from Table 1, the sports training mainly includes three vertical jumps, the take-off speed is 30 meters from the board, and then run 5 meters, and 100 meters, respectively, but there are great differences in the results of some events. These five qualified physical training indicators were selected as design factors for special athletes.

\subsection{CAD Is Assisted in the Establishment of Intelligent Technology}

5.2.1. Determination of the Network Structure. The basis of establishing CAD-assisted intelligent analysis model is to determine the structure of CAD-assisted intelligent analysis. Kolmogorov's theorem gives any continuous function in $[8,9]$; $f$ can be accurately realized by threelayer CAD-assisted intelligence technology. The input layer of the network is I neuron $f:[0,1]^{I} \longrightarrow R^{J}$ and the middle layer is $2 \mathrm{I}+1$ neuron. In this study, five design factors closely related to the athlete's special performance were selected, and five input neurons were set. According to Kolmogorov's theorem, a hidden layer was selected 

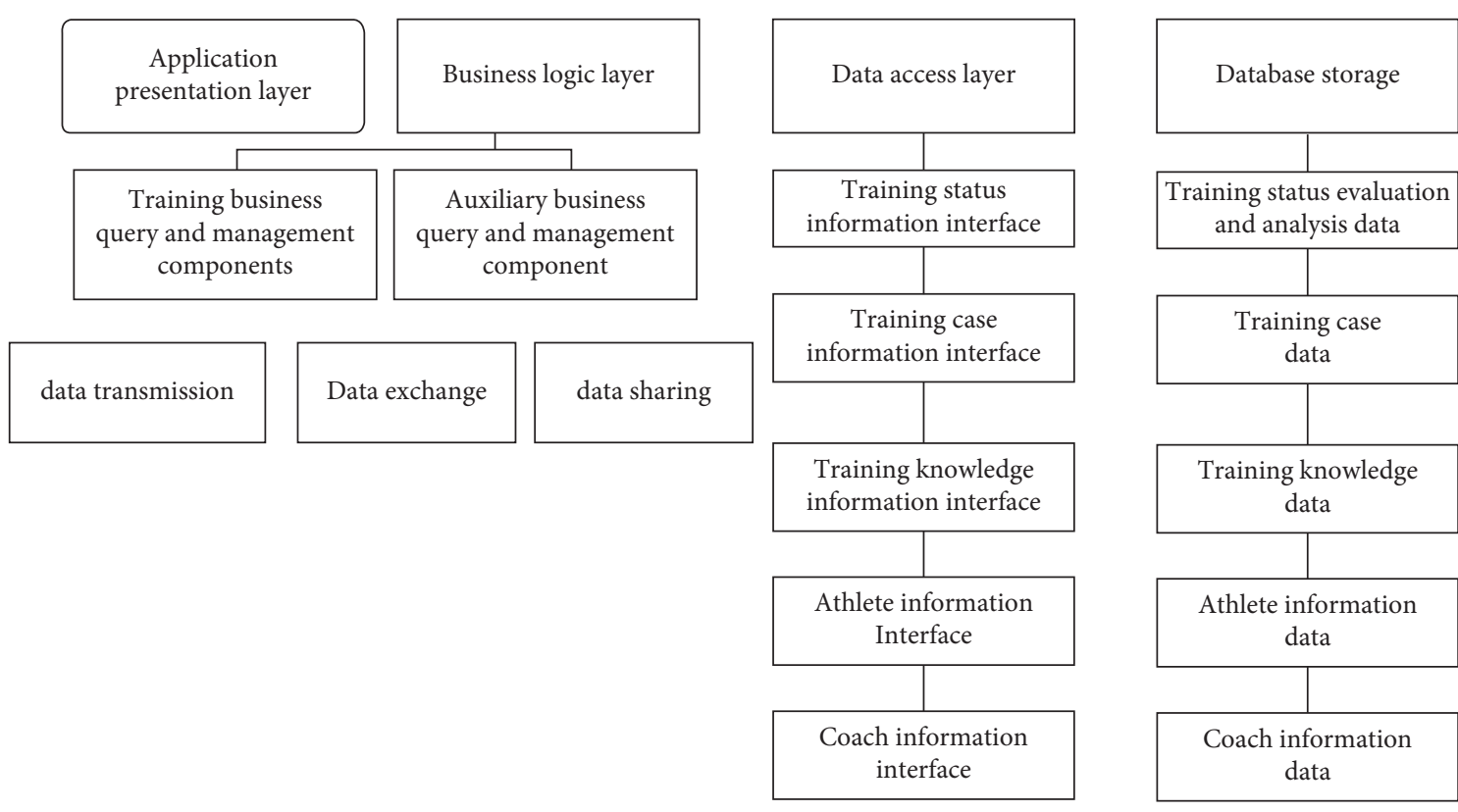

FIGURE 5: CAD auxiliary intelligent technology architecture.

TABLE 1: Correlation coefficient between quality sports training index and special long jump results.

Quality sports training indicators

Correlation

$100 \mathrm{~m}$ run $(\mathrm{S})$

The 30-meter run (S)

0.7501

$150 \mathrm{~m}$ run $(\mathrm{S})$

0.8004

Vertical triple jump (m)

1.0908

Last $5 \mathrm{~m}$ running speed $(\mathrm{m} / \mathrm{s})$

0.9394

Plate instant body center horizontal speed $(\mathrm{m} / \mathrm{s})$

0.6656

Wing leg swing speed $(\mathrm{m} / \mathrm{s})$

The center of gravity instantly rises from the board $(\mathrm{m} / \mathrm{s})$

0.7318

Squat barbell $(\mathrm{kg})$

TABLE 2: CAD assisted in intelligent analysis of input and output layer data.

\begin{tabular}{|c|c|c|c|c|c|c|}
\hline \multirow[b]{2}{*}{ Samples } & \multicolumn{5}{|c|}{ Enter the sample } & \multirow{2}{*}{$\begin{array}{l}\text { Output samples } \\
\text { Long jump } \\
\text { results (m) }\end{array}$} \\
\hline & $\begin{array}{l}\text { Vertical triple } \\
\text { jump }(\mathrm{m})\end{array}$ & $\begin{array}{l}\text { Last five speed of last } \\
\text { five speed }(\mathrm{s} / \mathrm{m})\end{array}$ & $\begin{array}{l}100 \mathrm{~m} \text { run } \\
(\mathrm{s})\end{array}$ & $\begin{array}{l}\text { The } 30 \text {-metre } \\
\text { run }(\mathrm{s})\end{array}$ & $\begin{array}{l}\text { The center of gravity instantly rises } \\
\text { from the board }(\mathrm{s} / \mathrm{m})\end{array}$ & \\
\hline 1 & 9.94 & 11.4 & 10.2 & 4.2 & 9.68 & 7.84 \\
\hline 2 & 10.22 & 10.87 & 9.7 & 3.8 & 9.70 & 7.75 \\
\hline 3 & 9.43 & 10.12 & 9.6 & 3.6 & 9.59 & 8.44 \\
\hline 4 & 9.89 & 10.24 & 8.8 & 3.9 & 9.67 & 8.03 \\
\hline 5 & 10.12 & 9.89 & 9.1 & 4.2 & 9.51 & 8.13 \\
\hline
\end{tabular}

and the number of neurons was set to 11 . The output layer is the score data of the specific item included.

5.2.2. Learning. The selected five quality sports training indicators and performance data of specific items were taken as sports training samples (see Table 2), five of the quality sports training indicators were input as design factors, and the results of corresponding specific items were taken as output data. Since CAD-assisted intelligence technology is most sensitive to $(0,1)$ due to the number between, the raw data need to be normalized and concentrated within the range of $(0,1)$. The specific treatment is given as follows: $x_{i}^{\prime}=x_{i}-x_{\min } / x_{\max }-x_{\min }$.

The minimum value $x_{\min }$ and the maximum value $x_{\max }$, respectively, indicate that $x_{i}^{\prime}$ is the normalized original value $x_{i}$. Input the normalized data into CAD-assisted intelligent technology to learn sports training samples, reduce the network output error to an acceptable level, and form the mapping between the quality indicators of sports training and the performance of specific events to achieve the 
accuracy of the performance of the special event of long jump design. The CAD-assisted intelligent analysis solves complex nonlinear problems, and the calculation process of learning is quite complicated. This study uses SPSS 19.0 statistical software to realize physical training and learning with CAD-assisted intelligent technology.

\subsubsection{Results of CAD-Assisted Intelligence Technology.} The data of quality sports training indicators from 2018 to 2019 were selected and first normalized, and then, the CAD auxiliary intelligent analysis mode with good sports training was replaced. Through software analysis and processing, the design value of specific project results was obtained. Quality sports training indicators and special results from 2018-2019 were fitted using least squares when performed using multiple linear regression patterns, and the mathematical model was obtained as follows:

$y=0.35 x_{1}+0.2 x_{2}-0.18 x_{3}-0.26 x_{4}+0.29 x_{5}+7.68$,

where $x_{1}, x_{2}, x_{3}, x_{4}$, and $x_{5}$ represent the previously selected quality sports training indicators, respectively. The long jumper's level 3 jump, 30 meters, the speed of the last five meters, 100 meters, and the center of gravity immediately leaving the springboard improved the initial speed.

Using the multilinear regression patterns, special performance design values for the 2018-2019 contestants were calculated using the above. Refer to Table 3, for the calculation results.

From the error column in Table 3, the design error of the CAD-assisted intelligence technology is much lower than that of the multilinear regression model. By calculating the error values of the various methods, the average relative error of the CAD-assisted intelligence technology was 0.046 and that of the multilinear regression model was 0.179 . The CAD-assisted intelligent analysis model was designed better than the multilinear regression model. The CAD auxiliary intelligence technology is more suitable for the special performance design of long jumpers.

Figures 6(a) 6(c) show the $X$-axis distance, $Y$-axis distance, and real-time analysis trajectory of the athlete's movement relative to the target position in the sports range. During this experiment, the search area for sports prediction is the range that athletes can move in this area, which is the so-called experimental platform. Searching all the current areas, we can find a moving image with a pixel size of $418 \times 415$. The Gaussian filter is used in this process, and the athlete position acquisition time takes about $60 \mathrm{~ms}$. Therefore, the control system cannot eliminate external interference, and the athlete cannot stabilize the original image, looking forward to the point.

From the analysis of the experimental results in Figure 7, it can be seen that the real-time analysis method of athlete posture is based on the analysis method, the real-time motion curve of the corresponding motion target position is in the $X$-axis direction and the error is in the $X$-axis direction, according to the motion curve, error value, and motion range in the $\mathrm{Y}$-axis direction in the motion area. The parameter values used in the experiment are $m=7, n=4$, and $b=0.4$. These three points are used as the base points of the historical athlete positions. The quartic curve fitting method is used to set the weighting coefficient in the searchable area of sports which is 0.4. In the sports analysis movement trajectory of the athlete in Figure 8, the physical line is mainly the sports trajectory corresponding to the target point reached by the $6 \mathrm{~s}$ athlete. Under human interference, the corresponding dashed line of $6 \mathrm{~s}$ athletes will use the sports trajectory that deviates from the balance point of the target trajectory after the athlete's human intervention. As shown in Figure 7, the numerical values used to analyze the accuracy of the athlete's motion have been ensured to ensure the robust performance of its sports analysis. If an athlete collides with an obstacle, the athlete's trajectory will also change, resulting in larger errors in the experiment process. Figures $7(\mathrm{~b})$ and $7(\mathrm{~d})$ can search for real-time motion analysis trajectory radius which is always greater than the analysis value, to ensure that the moving target cannot deviate. Figure 9 shows that the target images of frames 17, $23,49,72$, and 160 are the analyzed positions at the time corresponding to the black dots in the figure, and the black frame indicates the sports area. According to Figure 9, after obtaining the values, the maximum volume of part of the sports attitude will be larger than $1 / 6$ of the total sports area. Since the Gaussian filtering and the acquisition time of the athlete's position in the motion area takes about $10 \mathrm{~ms}$ or less, the calculation time for analyzing the CAD-assisted intelligent technology is $8 \mathrm{~ms}$. Therefore, it needs to be processed correctly, otherwise 100 frames/sec can ensure high-speed images, and you can get 100 frame/second image acquisition speed motion control system.

5.3. Analyze the Performance Comparison of CAD-Assisted Intelligent Technology. According to the analysis of the above experimental results, in the case of real-time analysis of sports, the time spent in real-time analysis is shorter than the time spent in image processing with this method. In this case, real-time analysis of sports can be quickly analyzed, which can highlight its advantages. The CAD-assisted intelligent technology used can also better complete the real-time analysis of sports. However, while completing the above two CAD-assisted intelligent technologies, the use of particle filter CAD-assisted intelligence technology can carry out single-structure, analytical sports, usually requiring a processing speed of about 15 frames per second.

Table 4 shows that, in the above technologies, the trajectory after collision with the edge is nonlinear, and the analysis error of the particle filter is obviously dominant, but the calculation time of the Kalman filter and the particle filter is long, and the frame frequency is $100 \mathrm{~Hz}$. Lost frame phenomenon CAD-assisted intelligent technology has the shortest calculation time, ensuring that the image processing reaches a frame rate of $100 \mathrm{~Hz}$. For sports balls with sports speeds greater than $1 \mathrm{~m} / \mathrm{s}$, the real-time analysis method based on CAD-assisted intelligent technology analysis is adopted, and the image sports area after sports analysis is equivalent to $1 / 4$ of all sports areas at most. 
Table 3: Preparation and accuracy of the design model.

\begin{tabular}{lcccc}
\hline \multirow{2}{*}{ No. } & \multirow{2}{*}{ Actual value $(\mathrm{m})$} & \multicolumn{2}{c}{ CAD-assisted intelligent technology } & \multicolumn{2}{c}{$\begin{array}{c}\text { Multiple linear regression model } \\
\text { Design value }(\mathrm{m})\end{array}$} \\
\hline 1 & & Design value $(\mathrm{m})$ & 0.09 & 8.36 \\
Error $(\mathrm{m})$
\end{tabular}

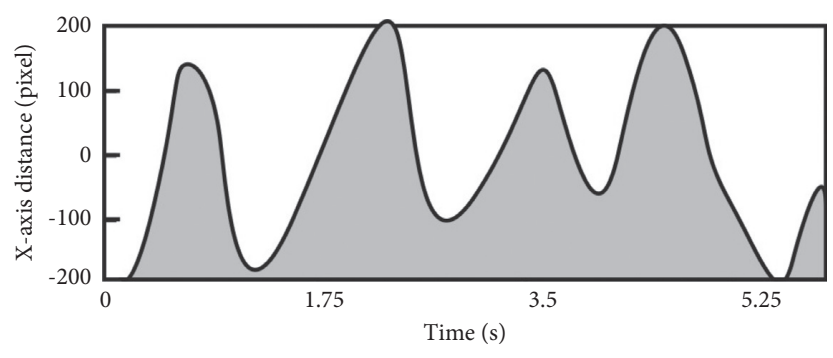

(a)

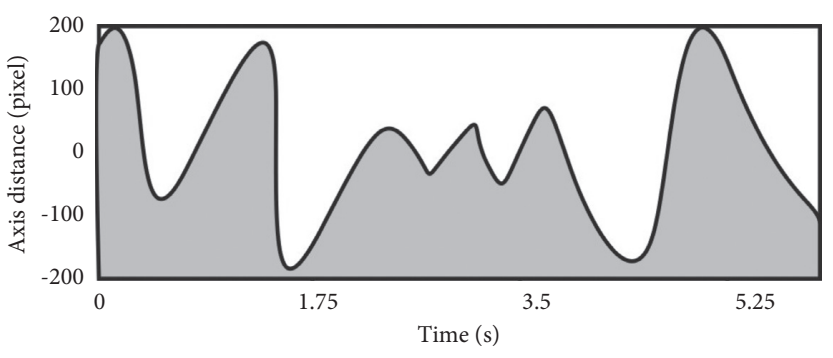

(b)

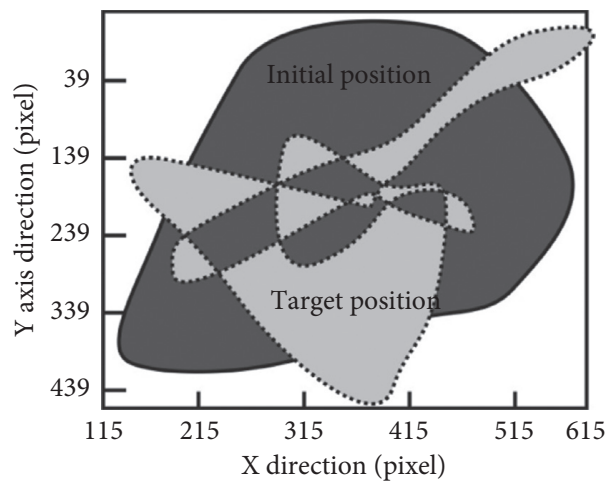

(c)

FIGURE 6: Search method of sports position in sports area.

5.4. Real-Time Location Analysis. Real-time posture analysis has gradually become an important research direction for in-depth analysis of sports, which can provide important reference value for sports training and competitive competitions. Due to the shortcomings of the existing real-time analysis methods of sports, this article is a real-time analysis method by constructing the sports goals of the knowledge city. Combine a variety of data for experimental analysis. In the real-time analysis experiment process of sports using CAD-assisted intelligent technology, the PC upper computer acquires the acquired moving images at a speed of $100 \mathrm{~Hz}$, that is, acquires each frame of images according to a cycle of $25 \mathrm{~ms}$. The analysis process of CADassisted intelligent technology in sports real-time analysis requires about $8 \mathrm{~ms}$. After the acquired sports images are processed, location information is extracted, and the moving target is analyzed, the image search is related to the size of the space in the area, and the space size of the sports area is $5 \mathrm{~ms}$ long, and the highest can reach $11 \mathrm{~ms}$. The position information obtained by real-time analysis of sports target motion will be fuzzy controlled by the DSP board to generate control instructions. The whole process takes about $1 \mathrm{~ms}$. The total time spent from acquiring a moving image to generating a motion command is $13 \mathrm{~ms}$ and $19 \mathrm{~ms}$, respectively. Quality inspection can ensure that the acquired position information of the moving target is completed within a $25 \mathrm{~ms}$ frame frequency cycle. The image processing process is based on the experimental hardware 

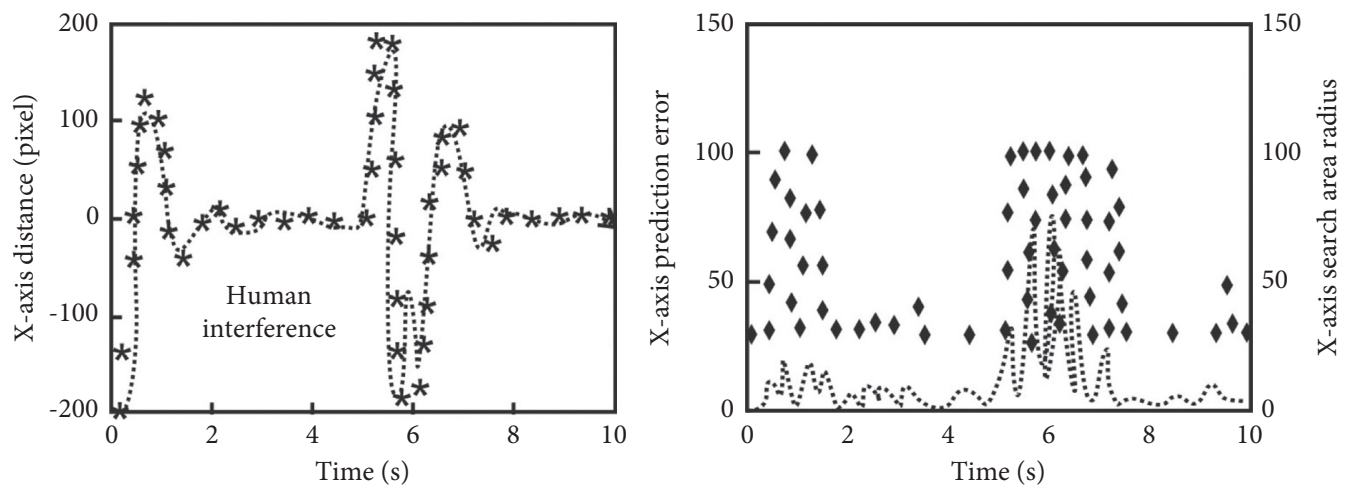

* Actual location Predicted location

- Search radius

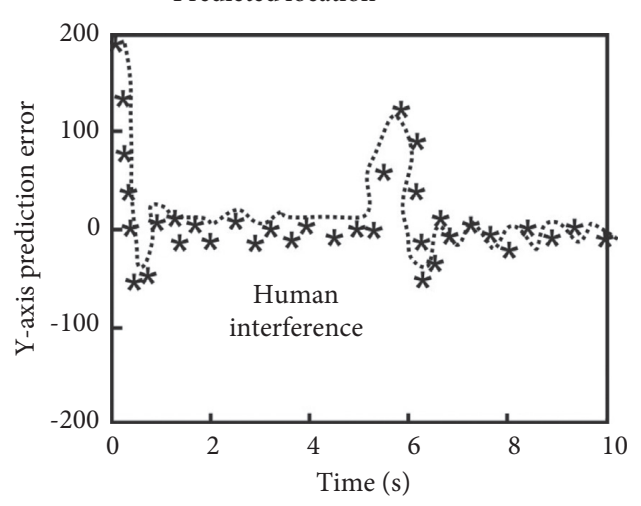
Prediction error

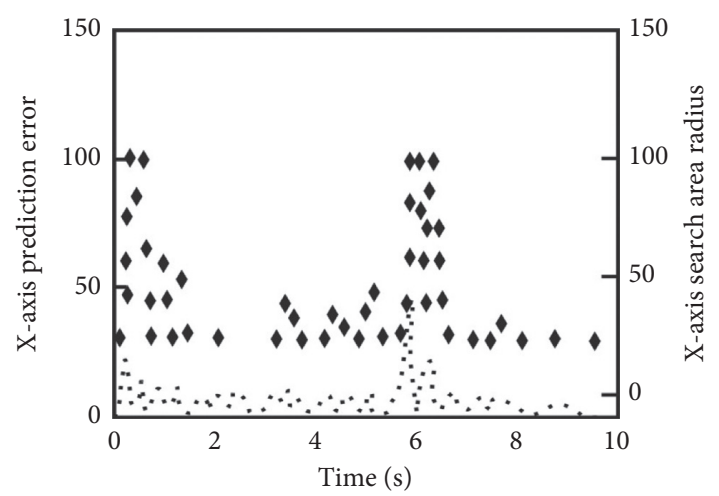

* Actual location

- Search radius Predicted location Prediction error

Figure 7: Real-time analysis performance of CAD-assisted intelligent technology athletes' posture.

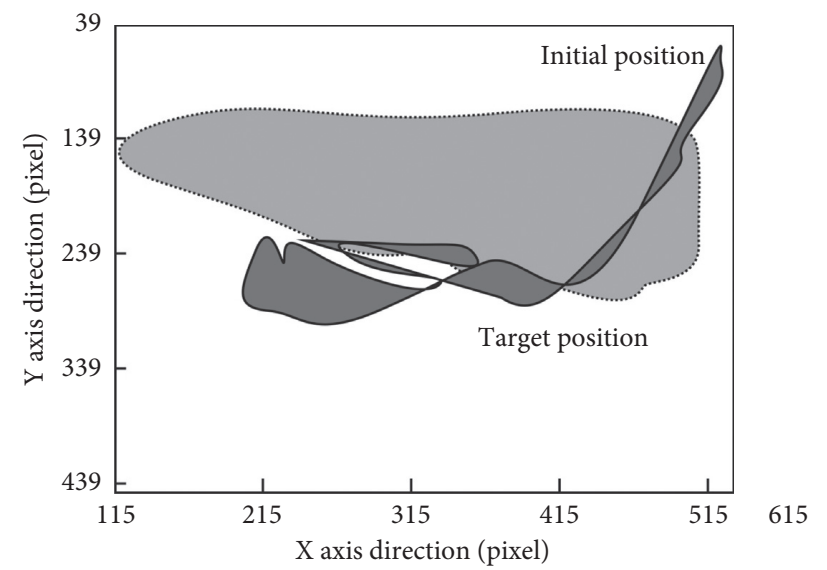

FIgURE 8: Athlete's sports trajectory under the real-time analysis method of posture with CAD-assisted intelligent technology. 


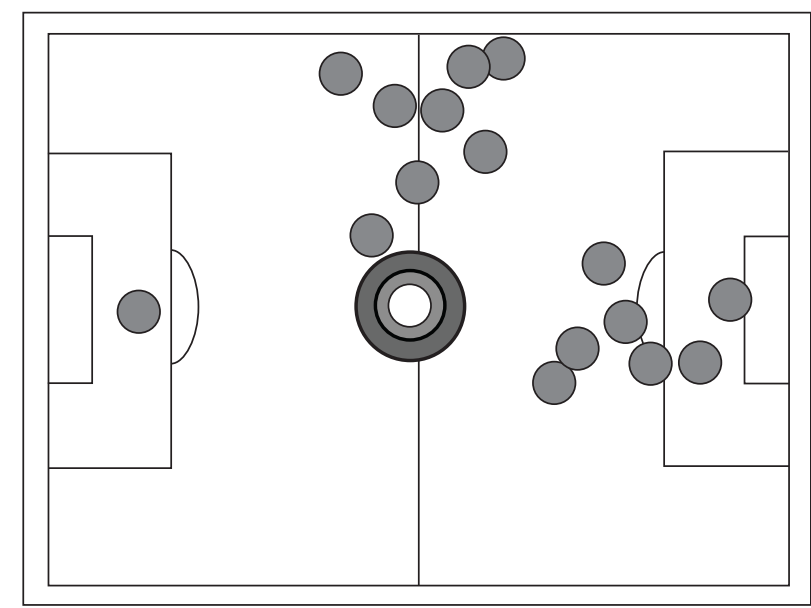

FIGURE 9: Real-time analysis of moving targets under CAD-assisted intelligent technology.

TABLE 4: Analyze the performance comparison of CAD-assisted intelligent technology.

\begin{tabular}{lccc}
\hline & CAD-assisted intelligent technology & Kalman filter & Particle filter \\
\hline Average calculation time (ms) & 3.214 & 38.347 & 131.967 \\
Average analysis error (pixel) & 7.231 & 6.454 & 4.332 \\
\hline
\end{tabular}

and CAD-assisted intelligent technology. According to the issued control instruction, this will make the wrong value replace the actual operation value and then realize the realtime positioning of the athlete's position.

\section{Conclusions}

From the perspective of the corresponding sports training methods and sports related knowledge, this paper mainly constructs the analysis model of the existing sports training projects and proposes a general design and framework of sports training using CAD auxiliary intelligent technology. The development and practical use of sports training system preliminarily analyze the effectiveness and feasibility of using CAD auxiliary intelligent technology in this paper, which can further effectively improve the formulation and effective implementation of sports training programs in sports and improve the effect and competitive level of sports training.

\section{Data Availability}

The labeled dataset used to support the findings of this study is available upon request to the author.

\section{Conflicts of Interest}

The author declares no conflicts of interest.

\section{Acknowledgments}

This study was sponsored by Jinling Institute of Technology.

\section{References}

[1] M. Dehghani, A. Delbar Saf, A. Vosoughi, G. Tebbenouri, and H. Ghazanfari Zarnagh, "Effectiveness of the mindfulnessacceptance-commitment-based approach on athletic performance and sports competition anxiety: a randomized clinical trial," Electronic Physician, vol. 10, no. 5, pp. 6749-6755, 2018.

[2] X. Leng, H. Jiang, X. Zou, and X. Zeng, "Motion feature quantization of athletic sports training based on fuzzy neural network theory," Cluster Computing, vol. 22, no. 2, pp. 4631-4638, 2019.

[3] Y. Jiang and Y. Wang, "Evaluation of teaching quality of public physical education in colleges based on the fuzzy evaluation theory," Journal of Computational and Theoretical Nanoscience, vol. 13, no. 12, pp. 9848-9851, 2016.

[4] T. Jindo, N. Kitano, K. Suzukawa, S. Sakamoto, and T. Nagamatsu, "Relationship of athletic sports with sense of coherence and mood states in male senior high school students: comparing athletes from a school soccer club and j-league youth teams," Bulletin of the Physical Fitness Research Institute, vol. 18, no. 1, pp. 1-9, 2018.

[5] B. W. Strafford, P. van der Steen, K. Davids, and J. A. Stone, "Parkour as a donor sport for athletic development in youth team sports: insights through an ecological dynamics lens," Sports Medicine - Open, vol. 4, no. 1, pp. 21-70, 2018.

[6] Y. Zhao, "Research on the diversified evaluation index system and evaluation model of physical education teaching in colleges and universities," Journal of Computational and Theoretical Nanoscience, vol. 14, no. 1, pp. 99-103, 2017.

[7] J.-H. Park and B.-K. Chun, "The influence of social support on self-determination and athletic performances of winter sports athlete," Korean Journal of Sports Science, vol. 26, no. 4, pp. 317-330, 2017.

[8] H. Huang and J. Chen, "Comprehensive evaluation of teaching quality of public physical education in colleges and universities," Kuram ve Uygulamada Egitim Bilimleri, vol. 49, no. 02, pp. 291-294, 2018. 
[9] Roos, G. Karen, and E. Wikstrom, "The epidemiology of lateral ligament complex ankle sprains in national collegiate athletic association sports," The American Journal of Sports Medicine, vol. 37, no. 5, p. 1, 2017.

[10] C. Clune, R. Boomsma, and R. Pucci, "The disparate roles of accounting in an amateur sports organisation: the case of logic assimilation in the gaelic athletic association," Accounting, Auditing \& Accountability Journal, vol. 191, no. 15, pp. 493508, 2019.

[11] Z. Liu, "Analysis of physical education evaluation system and design of intelligent evaluation system," Boletin Tecnico/ Technical Bulletin, vol. 55, no. 17, pp. 414-417, 2017.

[12] M. Roettger, "Modern sports dentistry," Textbooks in Contemporary Dentistry, pp. 7-21, 2018.

[13] J. Wallace, E. Beidler, and T. Covassin, "Assessment and management of sport-related concussion teaching trends in athletic training programs," Athletic Training Education Journal, vol. 13, no. 2, pp. 112-119, 2018.

[14] S. C. Budd and J. C. Egea, "General principles: caring for the athletic patient in sports dentistry," Springer International Publishing, vol. 9, no. 8, pp. 579-592, 2017.

[15] E. A. Bondareva and M. A. Negasheva, "Genetic aspects of athletic performance and sports selection," Biology Bulletin Reviews, vol. 7, no. 4, pp. 344-353, 2017. 